\title{
Post-Colonoscopy Recommendations After Inadequate Bowel Preparation: All in the Timing
}

\author{
Benjamin Lebwohl · Alfred I. Neugut
}

Received: 9 June 2013 / Accepted: 12 June 2013/Published online: 2 July 2013

(C) Springer Science+Business Media New York 2013

\begin{abstract}
Although colonoscopy effectively reduces colorectal cancer incidence and mortality [1,2], its effectiveness in the identification of adenomas and the prevention of colorectal cancer is critically dependent on the quality of the bowel preparation. Suboptimally prepared colons are associated with a longer cecal intubation time, a lower cecal intubation rate, and a lower polyp detection rate [3]. They also increase the total programmatic cost of colonoscopy due to the shortened repeat interval that is often recommended after such an examination [4].

Colonoscopy is imperfect even under ideal circumstances. A meta-analysis of studies of tandem colonoscopy yielded a pooled adenoma miss rate of $22 \%$ [5]. Fortunately, the highest reported miss rates concern the smallest lesions, which are the least likely to have advanced histologic features. Nevertheless, outside of these ideal circumstances, miss rates were higher in recent studies $[6,7]$. Two recent single-center studies quantifying adenoma miss rates based on repeated colonoscopies done shortly after suboptimally prepared examinations have yielded similar results with overall adenoma miss rates reported as 42 and $47 \%[6,7]$. On a per-patient basis, at least one adenoma was missed in 25 and $34 \%$ of these two cohorts. The miss rate for advanced lesions (previously quantified as $0-7 \%$ in tandem colonoscopy studies) [5] was $27 \%$ in one study
\end{abstract}

B. Lebwohl $(\varangle) \cdot$ A. I. Neugut

Department of Medicine, The Herbert Irving Comprehensive Cancer Center, College of Physicians and Surgeons, Columbia University, 180 Fort Washington Avenue, Suite 936, New York, NY 10032, USA

e-mail: bl114@columbia.edu

B. Lebwohl · A. I. Neugut

Department of Epidemiology, Mailman School of Public Health, Columbia University, New York, NY, USA
[6] with the other study reporting $18 \%$ for missed lesions that if identified would have changed the surveillance schedule [7].

Suboptimal bowel preparation is a common problem, occurring in $\sim 20 \%$ of all colonoscopies [8], with even higher rates among groups with lower socioeconomic status $[9,10]$. As such, our growing understanding of the deleterious impact of suboptimal bowel preparation has now made its way into clinical guidelines. Until recently, recommendations regarding timing of repeat colonoscopy were based solely on the number and histologic features of identified polyps, prefaced with a statement that such recommendations assume an optimal preparation [11]. The implication was that some examinations need to be repeated earlier, but that the precise timing was not specified.

The most recent statement, published by the US MultiSociety Task Force [12], states that examinations with a poor preparation should be repeated within 1 year, "in most cases." They also suggest that examinations deemed fair (but adequate to detect polyps larger than $5 \mathrm{~mm}$ ) should be repeated in 5 years, if small $(<10 \mathrm{~mm})$ adenomas were identified. While these recommendations are a welcome contrast to the previous vacuum, they do not offer concrete recommendations for common situations such as an examination with a fair preparation in which no adenomas were identified. Nor is it clear whether repeating an examination with a poor preparation "within 1 year" should be ideally done as soon as possible, or closer to the 1-year mark.

The study by Chokshi et al. [13] in this issue was conducted from 2004 to 2009, prior to the publication of guidelines addressing preparation quality. Nevertheless, the study provides practical information that informs our response to the commonly asked question: when should we repeat a colonoscopy with an inadequate preparation? In 
this study, the investigators reported colonoscopistrecommended follow-up intervals after an inadequate bowel preparation during screening colonoscopy at a single-center endoscopy suite. Consistent with prior survey studies, they reported that recommendations in this context are highly variable $[14,15]$, with a recommendation to repeat the next day in $\sim 5 \%$ of cases, with a similar proportion recommending repeat in 6-10 years. They also measured adherence to the recommendations, reporting that patients were more likely to comply with a next-day repeat colonoscopy than a recommendation for longer intervals. This is the first study to report the variability in recommended repeat colonoscopy intervals in clinical practice. Limitations include its single-center setting; the use of a subjective preparation quality scale (as opposed to the better-validated Boston Bowel Preparation score [16] or Ottawa score) [17]; a lack of reported polyp histology; and the potential for underestimating adherence for repeat colonoscopy performed outside of the reporting institution.

Despite these limitations, the results are striking and deserve our attention when considering the question of rescheduling patients with an inadequate bowel preparation. Among patients told to have their colonoscopy repeated the next day, nearly half (47\%) complied. Attendance rates for follow-up colonoscopy were significantly lower among patients instructed to follow-up, for example, 7-12 months later, with an adherence of only $15 \%$.

A next-day repeat colonoscopy intuitively seems more desirable than a repeat in 9 months. An inadequately prepared colon is still potentially a half-prepared colon, requiring only additional purgative to achieve an optimal preparation, rather than "starting from square one" at a later date. Moreover, the message to the patient told to return the next day is likely to be interpreted clearly: this examination was incomplete and "did not count," like any flawed test that needs repetition to be meaningful. In contrast, patients might interpret a mandate to return in 6 months as having been conferred some protection by the initial colonoscopy, and may be less eager to repeat it at such a short interval, especially if they had an initial expectation that this procedure is typically required every $5-10$ years.

Given the sample size of this study, it is premature to adopt routinely recommend next-day repeat procedures. Of the 17 told to return the next day, only eight (47\%) adhered. Although higher than adherence rates observed at longer intervals, given the small number sample size, a larger study is warranted. However, if these results do hold up, one can imagine such a policy taking shape: patients with a poor preparation should generally be rescheduled for a repeat examination the following day, whereas those with a fair preparation can be rescheduled at a longer (but truncated) interval.
In this study, nearly $22 \%$ of all colonoscopies were suboptimal, in line with the prior literature [8]. In the analysis of repeated examinations, only those patients whose preparations were deemed inadequate rather than suboptimal were considered, a much smaller number of patients (presumably, many of the patients with a suboptimal preparation had a quality deemed fair, but adequate). The frequency of inadequate preparation becomes relevant when contemplating a policy of routinely rescheduling next-day repeat examinations, an initiative that would require flexibility on the part of the endoscopy suite, endoscopist, and the patient.

The findings by Chokshi and colleagues suggest that rescheduling an inadequate colonoscopy the next day can often be achieved, and that patients are most likely to show up when it is rescheduled at this time. Future studies should involve the establishment of prospective protocols for the management of suboptimal preparation, and for the comparison of protocols with regard to outcomes such as procedure completion rate, adenoma detection rate, preparation quality on the follow-up examination, and the overall experience as rated by the patient.

Much progress has been made in recent years in improving bowel preparation quality. The practice of splitdosing is firmly established as effective [18]. Decreasing the "runway time" between the end of the purgative ingestion and the start of the colonoscopy is likewise an advance, especially regarding the ability to closely inspect the proximal colon [19]. While modifications of dosing schedule are made to improve bowel preparation, bowel preparation, regardless of quality, is rated by patients as the most difficult part of colonoscopy [20]. In order to maximize the effectiveness of colonoscopy in clinical practice, the rate of optimal preparations must improve, coupled with a plan of action dealing with suboptimal preparations that will provide the most effective disease prevention for the most patients.

\section{References}

1. Winawer SJ, Zauber AG, Ho MN, et al. Prevention of colorectal cancer by colonoscopic polypectomy. The National Polyp Study Workgroup. N Engl J Med. 1993;329:1977-1981.

2. Zauber AG, Winawer SJ, O'Brien MJ, et al. Colonoscopic polypectomy and long-term prevention of colorectal-cancer deaths. N Engl J Med. 2012;366:687-696.

3. Froehlich F, Wietlisbach V, Gonvers JJ, Burnand B, Vader JP. Impact of colonic cleansing on quality and diagnostic yield of colonoscopy: the European Panel of Appropriateness of Gastrointestinal Endoscopy European multicenter study. Gastrointest Endosc. 2005;61:378-384.

4. Rex DK, Imperiale TF, Latinovich DR, Bratcher LL. Impact of bowel preparation on efficiency and cost of colonoscopy. Am J Gastroenterol. 2002;97:1696-1700. 
5. van Rijn JC, Reitsma JB, Stoker J, Bossuyt PM, van Deventer SJ, Dekker E. Polyp miss rate determined by tandem colonoscopy: a systematic review. Am J Gastroenterol. 2006;101:343-350.

6. Lebwohl B, Kastrinos F, Glick M, Rosenbaum AJ, Wang T, Neugut AI. The impact of suboptimal bowel preparation on adenoma miss rates and the factors associated with early repeat colonoscopy. Gastrointest Endosc. 2011;73:1207-1214.

7. Chokshi RV, Hovis CE, Hollander T, Early DS, Wang JS. Prevalence of missed adenomas in patients with inadequate bowel preparation on screening colonoscopy. Gastrointest Endosc. 2012;75:1197-1203.

8. Harewood GC, Sharma VK, de Garmo P. Impact of colonoscopy preparation quality on detection of suspected colonic neoplasia. Gastrointest Endosc. 2003;58:76-79.

9. Kazarian ES, Carreira FS, Toribara NW, Denberg TD. Colonoscopy completion in a large safety net health care system. Clin Gastroenterol Hepatol. 2008;6:438-442.

10. Lebwohl B, Wang TC, Neugut AI. Socioeconomic and other predictors of colonoscopy preparation quality. Dig Dis Sci. 2010;55:7.

11. Levin B, Lieberman DA, McFarland B, et al. Screening and surveillance for the early detection of colorectal cancer and adenomatous polyps, 2008: a joint guideline from the American Cancer Society, the US Multi-Society Task Force on Colorectal Cancer, and the American College of Radiology. CA Cancer $J$ Clin. 2008;58:130-160.

12. Lieberman DA, Rex DK, Winawer SJ, et al. Guidelines for colonoscopy surveillance after screening and polypectomy: a consensus update by the US Multi-Society Task Force on colorectal cancer. Gastroenterology. 2012;143:844-857.

13. Chokshi RV, Hovis CE, Colditz GA, Early DS, Wang JS. Physician recommendations and patient adherence after inadequate bowel preparation on screening colonoscopy. Dig Dis Sci. (Epub ahead of print). doi:10.1007/s10620-013-2642-9.

14. Ben-Horin S, Bar-Meir S, Avidan B. The impact of colon cleanliness assessment on endoscopists' recommendations for follow-up colonoscopy. Am J Gastroenterol. 2007;102:2680-2685.

15. Hillyer GC, Basch CH, Lebwohl B, et al. Shortened surveillance intervals following suboptimal bowel preparation for colonoscopy: results of a national survey. Int J Colorectal Dis. 2013;28:73-81.

16. Lai EJ, Calderwood AH, Doros G, Fix OK, Jacobson BC. The Boston bowel preparation scale: a valid and reliable instrument for colonoscopy-oriented research. Gastrointest Endosc. 2009;69: $620-625$.

17. Rostom A, Jolicoeur E. Validation of a new scale for the assessment of bowel preparation quality. Gastrointest Endosc. 2004;59:482-486.

18. Kilgore TW, Abdinoor AA, Szary NM, et al. Bowel preparation with split-dose polyethylene glycol before colonoscopy: a metaanalysis of randomized controlled trials. Gastrointest Endosc. 2011;73:1240-1245.

19. Aisenberg J. Bowel preparation for colonoscopy: shortening the "runway time". Gastrointest Endosc. 2009;69:707-709.

20. Ko CW, Riffle S, Shapiro JA, et al. Incidence of minor complications and time lost from normal activities after screening or surveillance colonoscopy. Gastrointest Endosc. 2007;65:648-656. 